\title{
Comparative Analysis of Fatal Pedestrian Crashes between Kansas and USA
}

\author{
Uttara Roy \\ Department of Civil and Environmental Engineering, Lamar University, Beaumont, USA \\ Email: uroy@lamar.edu
}

How to cite this paper: Roy, U. (2019) Comparative Analysis of Fatal Pedestrian Crashes between Kansas and USA. Journal of Transportation Technologies, 9, 381-396. https://doi.org/10.4236/jtts.2019.93024

Received: June 30, 2019

Accepted: July 26, 2019

Published: July 29, 2019

Copyright (c) 2019 by author(s) and Scientific Research Publishing Inc. This work is licensed under the Creative Commons Attribution International License (CC BY 4.0).

http://creativecommons.org/licenses/by/4.0/

\begin{abstract}
Due to the increasing trend of population growth and urbanization, pedestrians form one of the largest single road user groups. However, they are the most neglected group among all road users. Pedestrian safety is now a growing concern in the USA. Identifying the factors associated with fatal pedestrian crashes plays a key role in developing efficient and effective strategies to enhance pedestrian safety. This study addresses safety issues by identifying contributory factors associated with fatal pedestrian crashes in Kansas and the USA. For Kansas, the study uses KARS (Kansas Accident Reporting System) database while for the USA FARS (Fatality Analysis Reporting System) database has been used. Different variables considered in this study are human variables (age, and gender), environmental variables (atmospheric condition and light condition), time (time of day, day of week, and crash month), location (intersection vs. mid-block), and roadway variables (speed limit). Different factors that are found to have an association with fatal pedestrian crashes are male pedestrians, older pedestrians, weekend, off peak hours, winter months, dark hours, non-intersection, clear atmospheric conditions, higher speed limit. The findings from Kansas have been compared with that from the USA. This study helps to implement potential countermeasures by identifying the factors that have an association with fatal pedestrian crashes.
\end{abstract}

\section{Keywords}

Fatal Crashes, Pedestrians, Chi-Square Statistics

\section{Introduction}

Due to the increasing trend of population growth and urbanization, pedestrians form one of the largest single road user groups. However, they are the most neglected group among all road users. The Federal Highway Administration 
(FWHA) conducted a survey in 2005, which is called Traveler Opinion and Perception (TOP) survey. According to the survey [1], about 107.4 million Americans use walking as a part of their daily trip. This turns out to be about $51 \%$ of the traveling public [1]. However, public policies in the United States have not done much to promote walking; rather it makes walking less feasible, less convenient, and more dangerous. The ease of crossing streets, the quality and spacing of pedestrian paths, sidewalk connectivity, topography, density, building orientation, and a mix of land uses at pedestrian scale are contributing factors to the pedestrian friendliness. Building a pedestrian friendly environment requires a lot of funding, too. Although federal funding for pedestrian facilities has increased somewhat over the past few decades, the funding is still a negligible percentage of total transportation funding.

Pedestrian fatalities are 36 times higher than car occupant fatalities per $\mathrm{km}$ traveled [2]. In the year 2008, pedestrians accounted for 4368 fatalities (11.75\%) of the total fatalities of 37,261 resulting from traffic accidents in the United States [3]. Therefore, building pedestrian facilities and providing access cannot be the complete solution, addressing safety issues should be done at the same time. Pedestrian safety is now a growing concern in the USA. Identifying the factors associated with fatal pedestrian crashes plays a key role in developing efficient and effective strategies to enhance pedestrian safety. This study addresses safety issues by identifying contributory factors associated with fatal pedestrian crashes.

The objectives of the study are:

- To compare the trend of pedestrian fatalities in Kansas to that of the USA,

- To identify the causative factors of fatal pedestrian crashes in Kansas,

- To compare the findings with USA,

- To determine if any relationship exists between different variables considered with the help of Chi-Squared test.

The remainder of the paper is organized as follows: Section 2 describes the relevant studies on pedestrian crashes, Section 3 describes the data and the methodology used in this study, Section 4 analyzes the results and the statistical analysis used in this study, Section 5 concludes the study.

\section{Literature Review}

A study done in Florida by Spainhour et al. [4] found out that the primary contributing cause of $76 \%$ of the pedestrian crashes (the study reviewed 353 fatal pedestrian crashes in Florida) is some sort of pedestrian behavior. Alcohol consumption either by the pedestrian or by the driver and dark conditions or insufficient lighting are responsible for about $45 \%$ and $60 \%$ of pedestrian crashes respectively. The study also found that around $15 \%$ of the pedestrian crashes resulted due to limited access facilities (interstate, toll road, and other limited access facilities).

The largest risk factors for fatal injuries of pedestrians hit by a vehicle are identified by Siddiqui et al. [5] in a study done in Florida. According to the 
study, the largest risk factors in a decreasing order are: being at least 65 years old, being hit by a driver who is DUI, being involved in a crash on the US road system, walking in foggy conditions, being WUI, being hit by a driver with physical disabilities, and being hit by large vehicles. The study concluded that if other factors remain constant, once involved in a crash, there is a greater fatality risk for elderly pedestrians (5.8\% higher) than pedestrians aged from 25 through 64. The probability of a pedestrian being killed when struck by a vehicle is higher in midblock locations than at intersections for any light conditions. However, light conditions have a greater effect on pedestrian fatalities than locations. The study also found out that daylight reduces the fatal injuries both at midblock locations and at intersections.

Another study conducted by Carter et al. [6], which is focused on rural highways, suggests that the percentage of pedestrian fatalities is higher ( $8 \%$ higher) in rural areas than in urban areas. The study also concluded that $82 \%$ of pedestrian crashes in rural areas occur at non-intersection locations. About 50\% of total pedestrian crashes occur in dark hours for rural areas whereas around 54\% occur in daylight for urban areas.

There was a research at Monash University Accident Research center carried out by Corben et al. [7] on fatal pedestrian crashes and the study developed a method for estimating the relative risk of a fatal pedestrian crash as a function of travel speed. The study concluded that pedestrian fatalities are strongly related with driver speed choice and the small reductions of speed $(5-10 \mathrm{~km} / \mathrm{h})$ can reduce the risk of a pedestrian death by almost $80 \%$.

Despite significant research efforts made in past studies, to the best of author's knowledge, there was no study done which compared the factors associated with fatal pedestrian crashes between Kansas and USA. The factors that have been found to have strong association with fatal pedestrian crashes will be helpful to implement countermeasures which will ultimately reduce the number of fatal pedestrian crashes.

\section{Data and Methodology}

The study uses KARS (Kansas Accident Reporting System) database [8] to extract the number of fatal pedestrian crashes for Kansas from the year 1999 to 2008. FARS (Fatality Analysis Reporting System) database [9] is used to get the number of fatal pedestrian crashes for the USA. Publication from FARS and query are also used in this study. Different variables that are used in this study are human variables (age, and gender), environmental variables (atmospheric condition, and light condition), time (time of day, day of week, and crash month), and speed limit.

To analyze the data, chi-squared statistics have been used. Degree of freedom has been referred to as the number of independent variables which make up the statistics [10]. If $\mathrm{k}$ is the number of independent constraints in a set of data of $\mathrm{n}$ observations, then degree of freedom will be $n-k$. Thus, in a set of $\mathrm{n}$ observations, the degree of freedom for $\chi^{2}$ are $n-1[10]$. Also, if any of the population 
parameter is calculated from the given data and used for computing the expected frequency, then it is necessary to subtract one degree of freedom for each parameter calculated in order to estimate $\chi^{2}$-test of goodness of fit. Therefore, if $s$ is the number of populations parameter estimated from the sample observation of $n$ number, then the degree of freedom for $\chi^{2}$ would be (n-s-1) [10]. In case one or more of the theoretical frequencies are less than 5 , then it is also necessary to subtract the degrees of freedom lost in pooling these frequencies with the preceding or succeeding frequency while applying $\chi^{2}$-test. So, in a $\left(r^{\star} s\right)$ contingency table, the required number of degrees of freedom can be expressed as follows [10]:

$$
\text { Degree of freedom }=r s-(r+s-1)=(r-1)(s-1)
$$

Chi-squared test has been formulated by Karl Pearson in 1900 and it is a very powerful test for testing significance of the discrepancy between theory and experiment [10]. The idea behind the hypothesis test is that if $\mathrm{H}_{0}$ is true, then the observed and expected values are likely to be close to each other. To define the test statistic, let $k$ be the number of outcomes and let $O_{i}$ and $E_{i}$ be the observed and expected number of trials, respectively, that result in outcomes $i$. So, the chi-squared statistic is [11]

$$
\chi^{2}=\sum_{i=1}^{k} \frac{\left(O_{i}-E_{i}\right)^{2}}{E_{i}}
$$

It is called chi-square distribution with $(k-1)$ degrees of freedom. The larger the value of $\chi^{2}$ is, the stronger the evidence against $H_{0}$. It is necessary to know the null distribution of this test statistic in order to determine the p-value for the test.

\section{Analysis}

For trend analysis, ten years' crash data from 1999 to 2008 have been considered both for USA and Kansas. However, when different factors are considered which have association with fatal pedestrian crashes, data for 2004-2008 have been considered. For statistical analysis also, data for USA from 2004-2008 have been considered. For Kansas, the number of observations is low and that is why, statistical test was not considered.

\subsection{Trends of Pedestrian Fatalities in Kansas and USA}

Figure 1 shows the number of total fatalities, pedestrian fatalities and percentage of pedestrian fatalities to that of total fatalities in USA and Kansas from the year 1999 to 2008. From Figure 1, it is found that pedestrian fatalities do not constitute a significant portion of total fatalities; rather the percentage of pedestrian fatalities to that of total fatalities do not exceed $12 \%$ and $6 \%$ for USA and Kansas respectively. Throughout the 10 years, there have been fluctuations in the trends of percentage of pedestrian fatalities both for Kansas and USA. However, the trends do not demonstrate the fact that pedestrian safety issues either in Kansas 


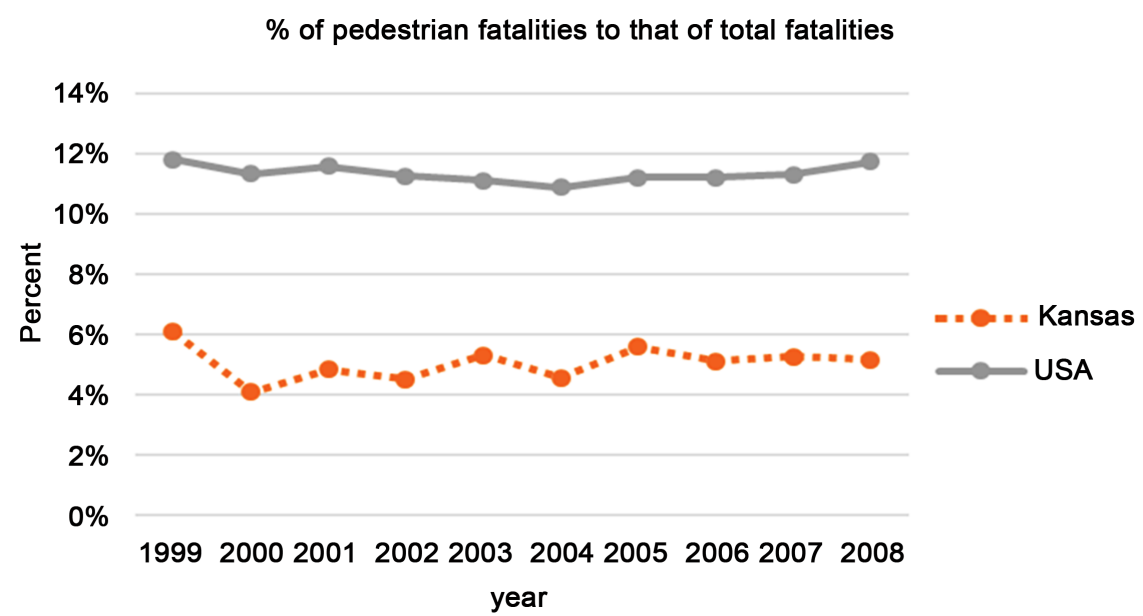

Figure 1. Percentage of pedestrian fatalities to that of total fatalities in Kansas and USA [3].

or in USA have been improved. On the contrary, a study conducted by the $\mathrm{Na}$ tional Household Travel Survey (NHTS) in 2001 explains the fact that in the United States, travel behavior is accompanied mainly by auto and in recent decades, auto ridership has been increased tremendously (from 66.9\% in 1960 to $87.9 \%$ in 2000) [12]. On the other hand, the percentage of trips made by public transit and walking has been decreased enormously. The share of walking for journey to work in 1960 was $10.3 \%$ and the percentage has been decreased to $2.9 \%$ in 2000 [12].

Table 1 displays the modal share of different modes of transport for Journey-to-Work in the United States from the year 1960 to 2000.

Therefore, as level of exposure for pedestrians has been decreased drastically, number of pedestrian fatalities has been less too.

\subsection{Involved Persons in Fatal Pedestrian Crashes}

Gender and age of persons who involved in fatal pedestrian crashes are analyzed in this section.

\subsubsection{Gender}

Figure 2 elucidates that for every year analyzed, male pedestrians constitute the larger portion of total pedestrian fatalities than females. In 2007, there is no female pedestrian fatality in Kansas (there is a small percentage of fatalities in 2007 for which gender was not able to determine) and for rest of the years being analyzed; the percentage of male pedestrian fatalities is more than $65 \%$. If the findings are compared to USA, the same trend is obtained (Figure 3). The reason might be the exposure level as males are more involved with economic activities than females which might lead to higher exposure as well as higher percentage of fatal crashes for males. Some other issues might also contribute to larger number of fatal pedestrian crashes for males such as aggressiveness, and less inclination of following traffic rules and regulations etc. 


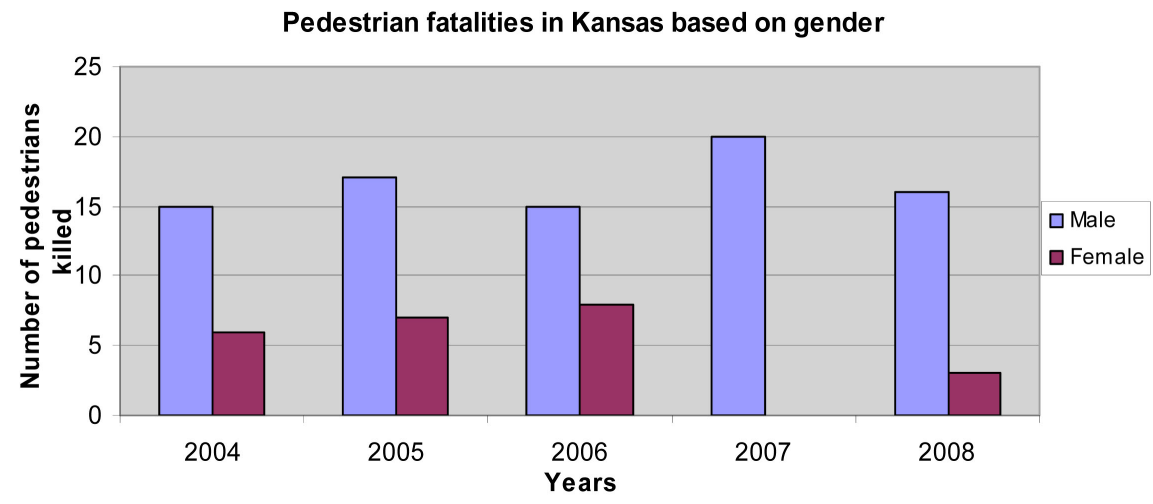

Figure 2. Categorization of pedestrian fatalities in Kansas based on gender.

\section{Pedestrian fatalities based on gender}

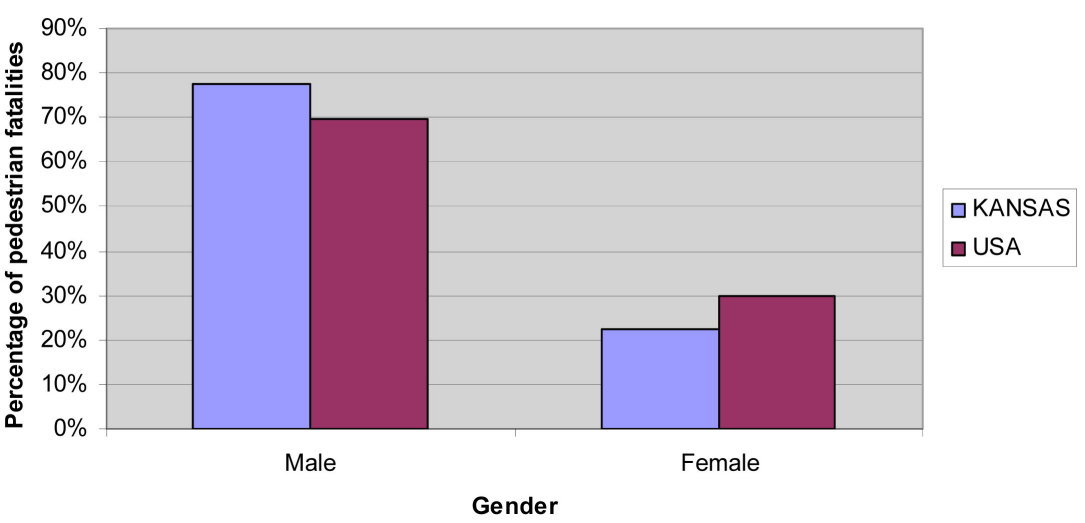

Figure 3. Comparison of pedestrian fatalities (based on gender) between Kansas and USA.

Table 1. Trends in modal split for the journey-to-work in the United States (1960-2000) [12].

\begin{tabular}{cccccc}
\hline Mode of & \multicolumn{5}{c}{ Census Year } \\
\cline { 2 - 6 } Transportation & 1960 & 1970 & 1980 & 1990 & 2000 \\
\hline Auto & 66.9 & 77.7 & 84.1 & 86.5 & 87.9 \\
Public Transit & 12.6 & 8.9 & 6.4 & 5.3 & 4.7 \\
Walk & 10.3 & 7.4 & 5.6 & 3.9 & 2.9 \\
Bicycle & $\mathrm{Na}$ & $\mathrm{Na}$ & 0.5 & 0.4 & 0.4 \\
All & 100 & 100 & 100 & 100 & 100 \\
\hline
\end{tabular}

${ }^{*}$ The modal share doesn't add to 100 because there is a small percentage of other modes (work at home etc.) which is not included here.

\subsubsection{Age}

If age group is considered, it is found that age groups of 45 - 54 and $65+$ years are the most vulnerable groups. For Kansas, the maximum number of pedestrian fatalities was found for the 65+ years older pedestrians in 2004 (Figure 4). For the age groups of 25 - 34 and for pedestrians less than 16 years old, the number of pedestrian fatalities is the lowest. 
Pedestrian fatalities in Kansas based on age

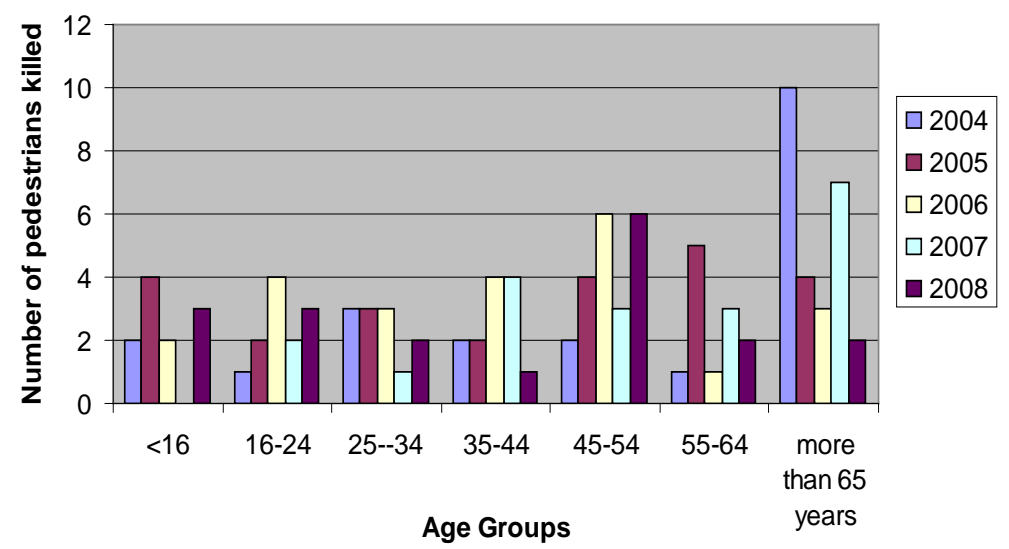

Figure 4. Categorization of pedestrian fatalities in Kansas based on age.

Age groups of 25 - 34 and 55 - 64 comprise of the lesser number of pedestrian fatalities too. Figure 5 which compares the findings with USA reveals the same fact. The reason might be as people get older, their ability to respond to critical situations have decreased. As a result of natural aging, older people experience physical difficulties such as loss of vision, slower perception reaction times, and deterioration of physical strength, all of which might lead to larger number of fatal pedestrian crashes for them.

\subsection{Time of Occurrence of Fatal Pedestrian Crashes}

For time of occurrence, three variables are considered: days of week, time of day and crash month. These three variables are analyzed in this section.

\subsubsection{Days of Week}

Figure 6 and Figure 7 explain the fact that most of the fatal pedestrian crashes occur on Friday and Saturday. The number of fatal crashes is the lowest on Sunday. Other weekdays also do not contribute to the significant number of fatal pedestrian crashes compared to Friday and Saturday.

The reason might be the increased level of interaction between motorized vehicles and pedestrians during these two days. Also, alcohol could be another reason for which there is a larger number of fatal pedestrian crashes during weekends. One exception to this is that in 2005 the maximum number of fatal crashes occurs on Tuesday in Kansas. The finding is compared with USA and the same information is found. This is illustrated by Figure 7 .

\subsubsection{Time of the Day}

If the time of the day is considered, then it is found that off-peak hours (6 pm to $6 \mathrm{am}$ ) are the critical time for fatal pedestrian crashes to occur (Figure 8).

The maximum number of fatal crashes occurs between midnight and 6 am in the morning (24\%) followed by between 9 pm and 12 pm (23\%). The least number of pedestrian fatalities occur between 6 am and 9 am in the morning (12\%). For USA this time interval accounts only for $9 \%$ of the total fatalities which is 


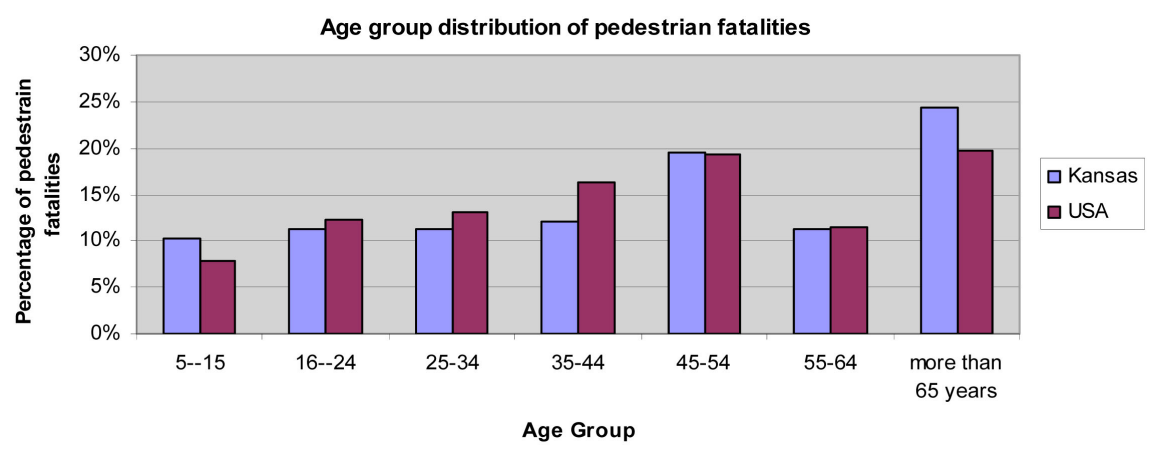

Figure 5. Comparison of pedestrian fatalities (based on age) between Kansas and USA.

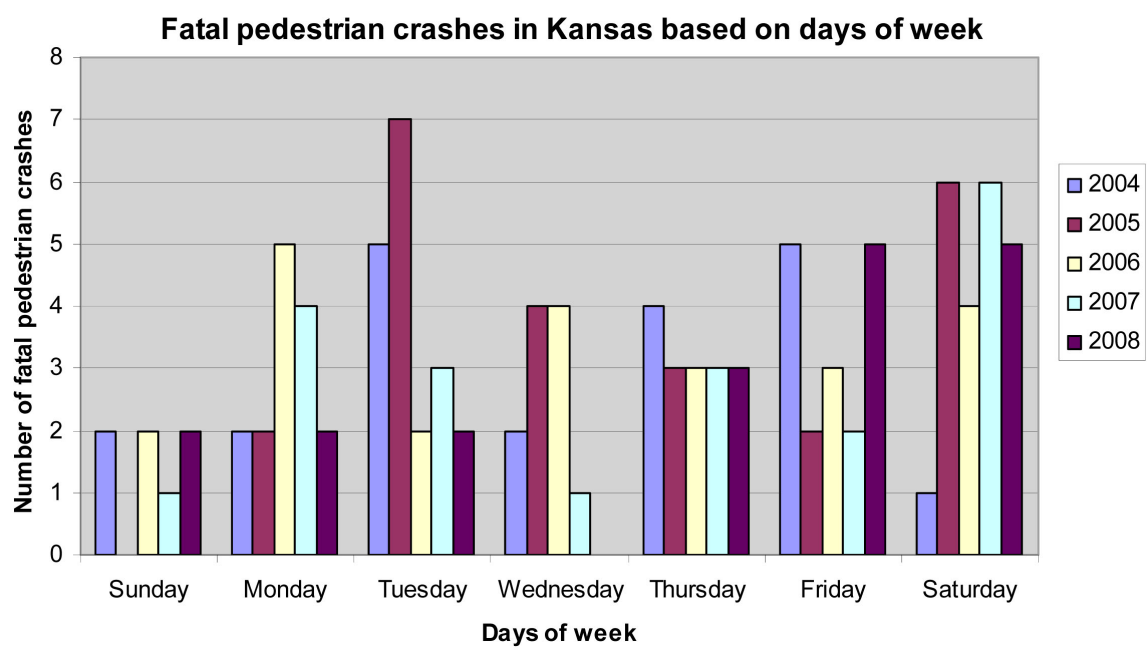

Figure 6. Categorization of fatal crashes in Kansas based on days of week.

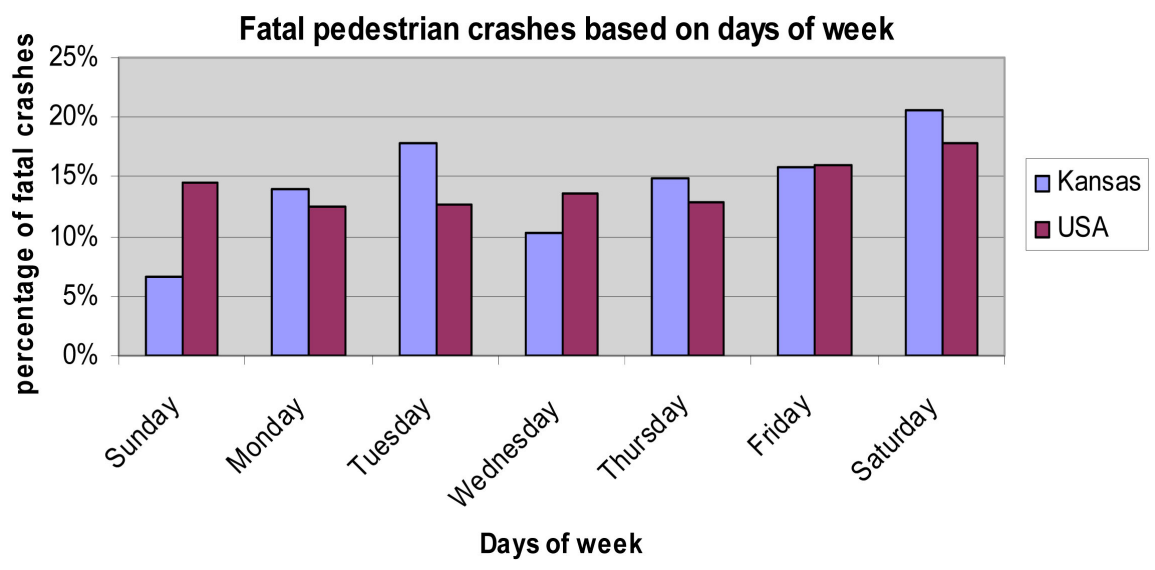

Figure 7. Comparison of fatal pedestrian crashes (based on days of week) between Kansas and USA.

the lowest number of pedestrian fatalities among all time intervals (Figure 9). The highest number of pedestrian fatalities for USA occurs between $6 \mathrm{pm}$ and 9 pm (25\%). Lack of visibility after $6 \mathrm{pm}$ because of darkness as well as higher exposure might be the factors leading to a higher number of fatal pedestrian crashes during that time period. 


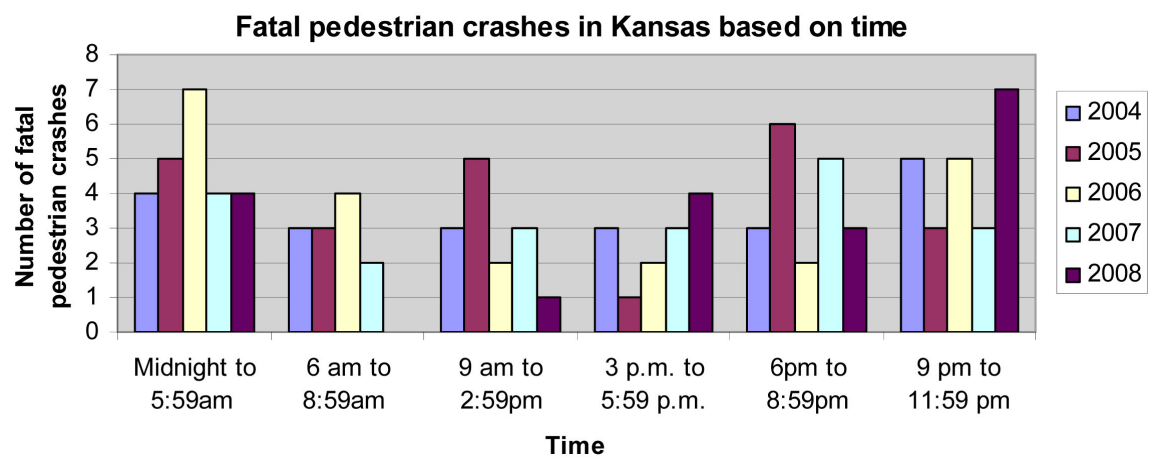

Figure 8. Categorization of fatal crashes in Kansas based on times of day.

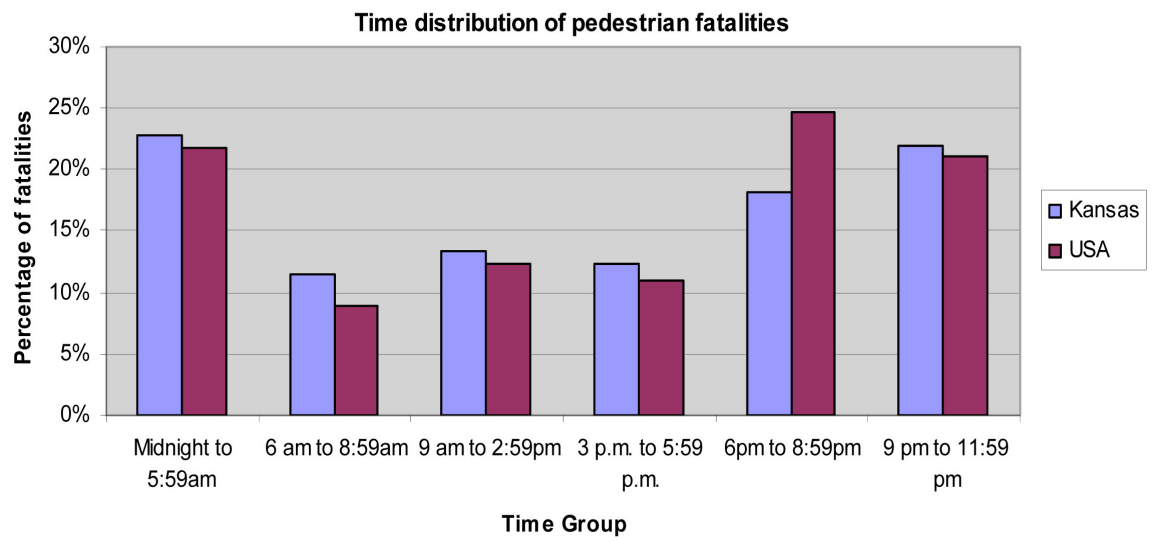

Figure 9. Comparison of fatal pedestrian crashes between Kansas and USA based on time of the day.

\subsubsection{Crash Month}

Fatal pedestrian crashes occur in all months, but some months or some particular seasons are critical for crashes to take place. Figure 10 demonstrates that for USA, October, November and December $(10.06 \%, 10.04 \%$, and $10.05 \%$ respectively) are the prime months for fatal crashes to take place. The maximum number of crashes in Kansas is in December (18\%). March and August are also critical months for fatal crashes to take place in Kansas; the proportions of fatal crashes are $14 \%$ and $12 \%$ respectively.

\subsection{Contribution of Environmental Variables to Fatal Pedestrian Crashes}

\subsubsection{Atmospheric Condition}

When atmospheric condition is considered, it is found that the majority of fatal crashes occur on a clear day with no adverse condition (Figure 11). The percentages of crashes that occur on a clear day are $87 \%$ of total fatal pedestrian crashes for Kansas and $89 \%$ for USA. Rain comprises of $10 \%$ of total fatal pedestrian crashes for Kansas and $8 \%$ for USA. Snow contributes to the least number of crashes both for the USA and Kansas. This is explained by the facts that when atmospheric conditions are good, people tend to go out for a walk which contributes to the higher number of fatal pedestrian crashes. 
$\%$ of fatal pedestrian crashes based on month

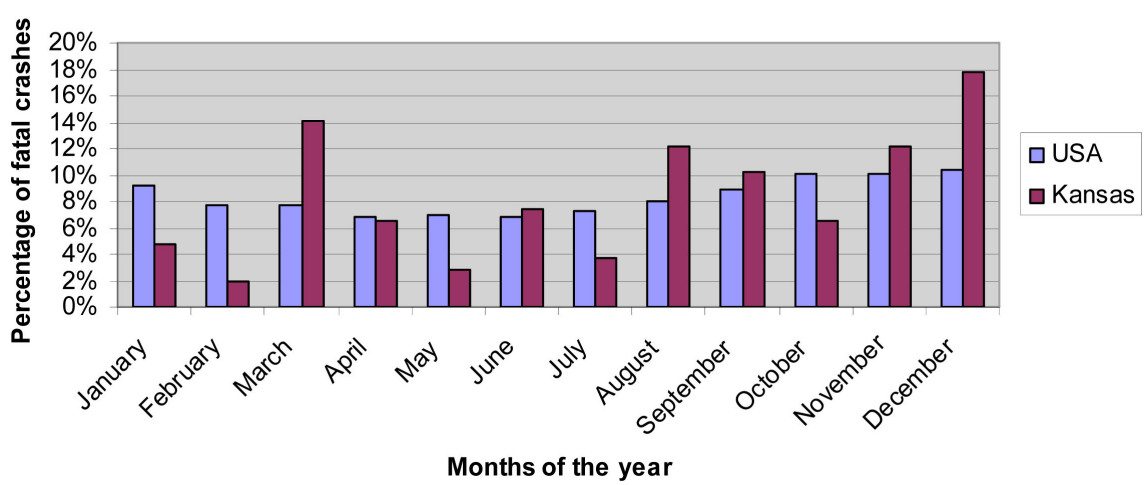

Figure 10. Comparison of fatal pedestrian crashes between Kansas and USA.

Fatal pedestrian crashes based on atmospheric condition

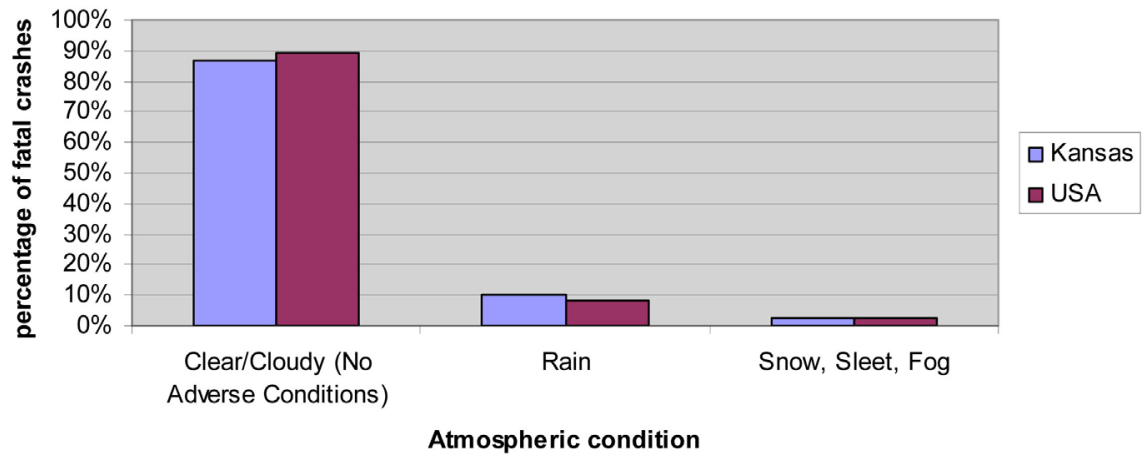

Figure 11. Comparison of fatal pedestrian crashes between Kansas and USA based on atmospheric condition.

\subsubsection{Light Condition}

When light condition is considered, it is found that most of the fatal pedestrian crashes occur in dark, with or without light (Figure 12). In 2006, 11 fatal crashes occur in Kansas when it was dark without light. This is the maximum number of fatal crashes when light condition is considered in Kansas throughout the whole analysis period. When the finding for Kansas is compared with the USA, the same information is obtained (Figure 13). Both the USA and Kansas have almost the same percentage of crashes (29\%) in daylight condition. Therefore, both dark condition and dark with light represent about of $71 \%$ of total crashes both for Kansas and USA. This can be explained by the fact that lack of visibility during dark condition leads to larger number of fatal pedestrian crashes.

\subsection{Location of Fatal Pedestrian Crashes}

If location of fatal pedestrian crashes is considered, it is found that most of the fatal pedestrian crashes occur in non-intersection or in other words, in mid-block. The same trend is found for both Kansas and the USA. $79 \%$ of the crashes occur in Kansas is in non-intersection and for the USA the percentage is about $74 \%$ (Figure 14). 


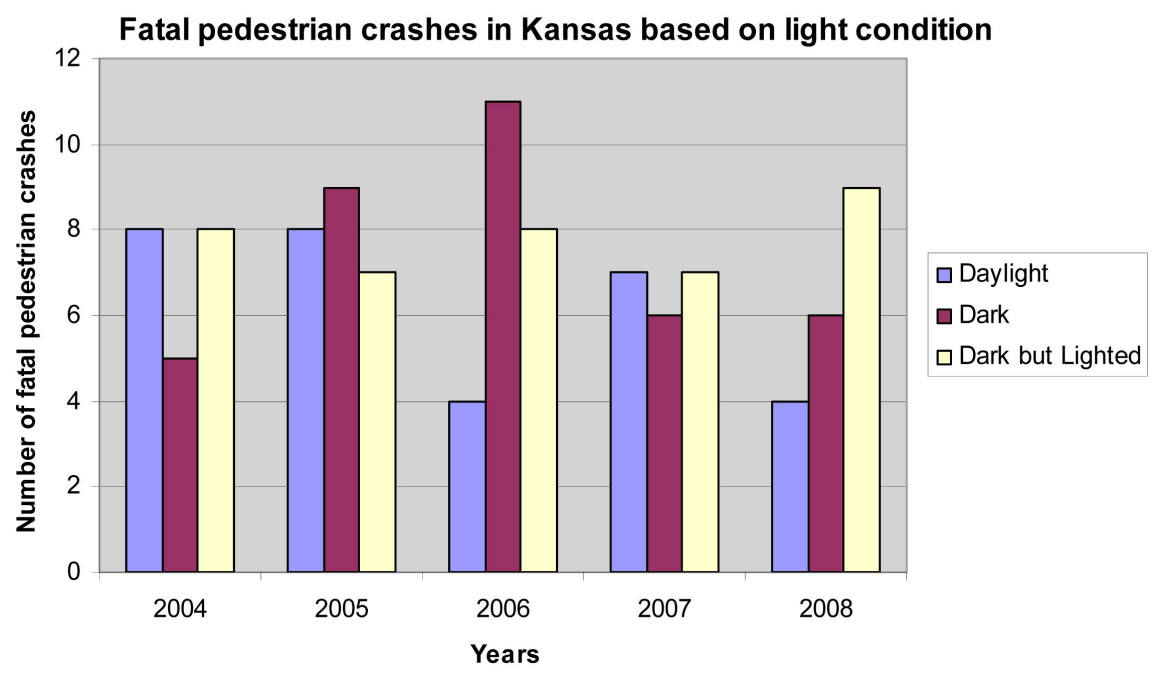

Figure 12. Distribution of fatal pedestrian crashes in Kansas based on light condition.

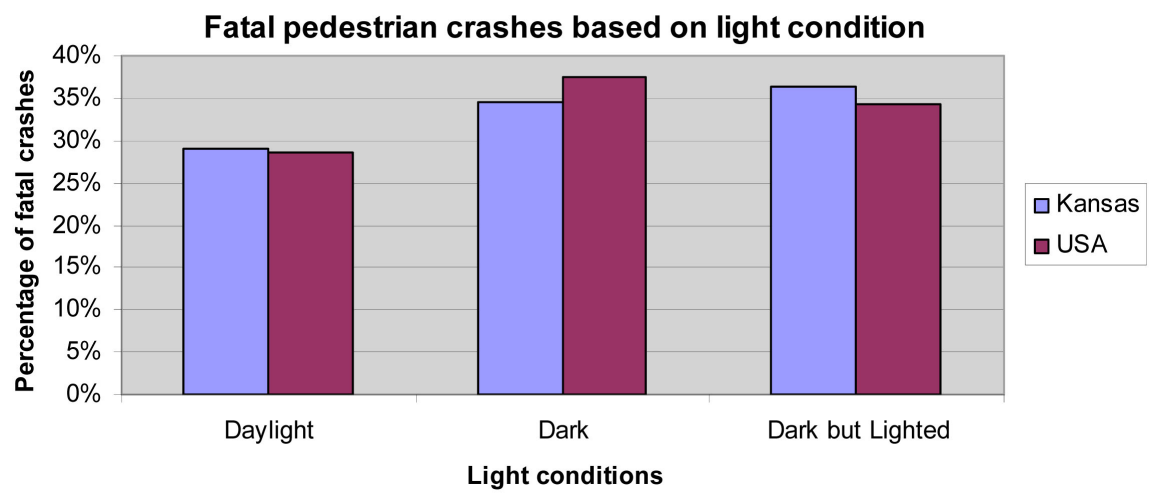

Figure 13. Comparison of fatal pedestrian crashes between Kansas and USA based on light condition.

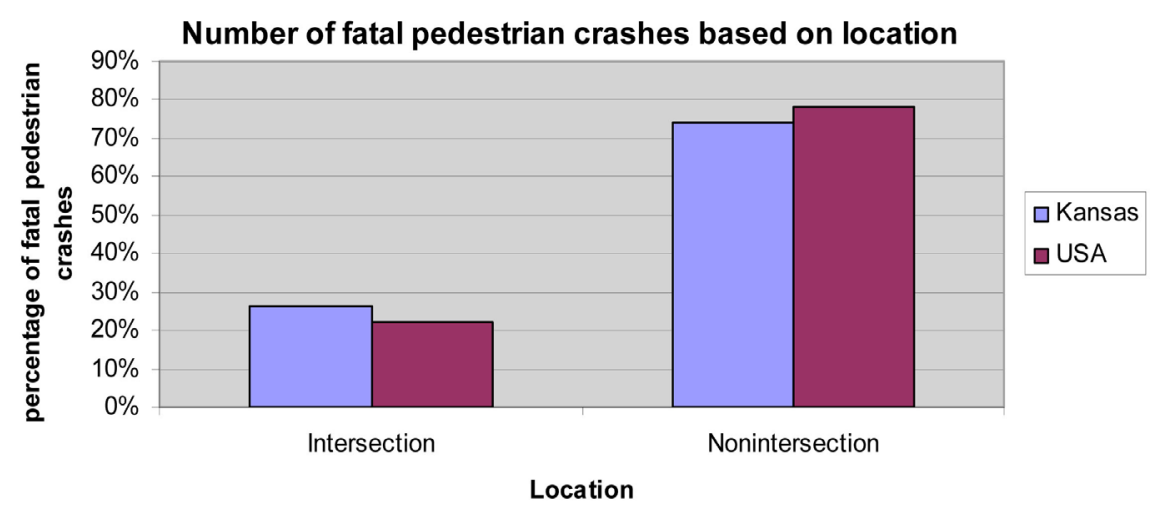

Figure 14. Comparison of fatal pedestrian crashes between Kansas and USA based on location.

\subsection{Contribution of Speed Limit to Fatal Pedestrian Crashes}

When speed limit is considered, different trends are found for Kansas and the USA (Figure 15). For Kansas, it is discovered that speed limit of about $60 \mathrm{mph}$ contribute to the highest number of fatal crashes. On the other hand, for the 


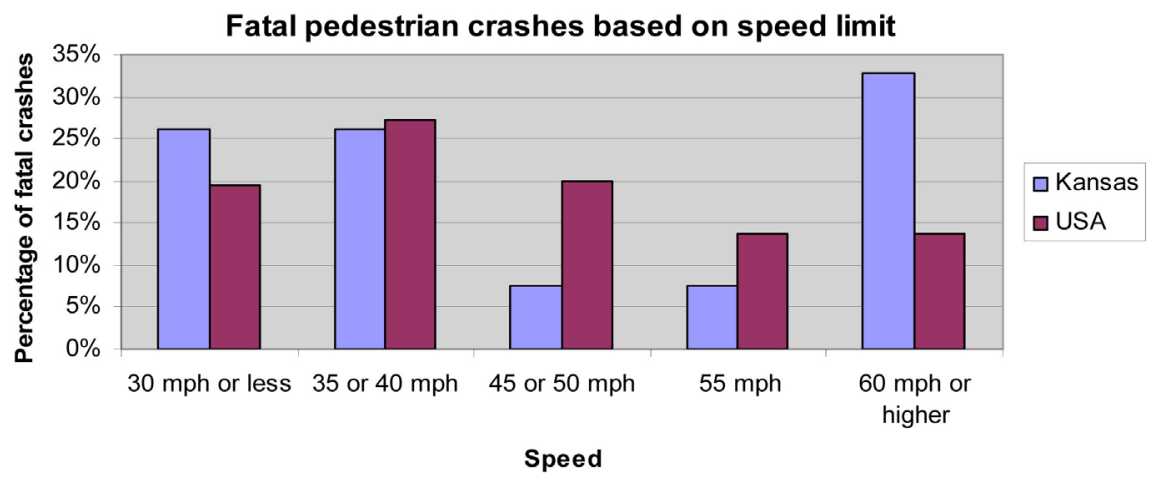

Figure 15. Comparison of fatal pedestrian crashes between Kansas and USA based on light condition.

USA speed limit between $35 \mathrm{mph}$ and $40 \mathrm{mph}$ represent the maximum number of crashes (27\%). For Kansas, speed limits between $30 \mathrm{mph}$ and $40 \mathrm{mph}$ account for $52 \%$ of total crashes $(26 \%$ crashes for $30 \mathrm{mph}$ and $26 \%$ for $35 \mathrm{mph}$ or 40 $\mathrm{mph}$ ), whereas the same speed limit represent $20 \%$ and $27 \%$ of total crashes. Speed limit between $45 \mathrm{mph}$ and $55 \mathrm{mph}$ represent a very small percentage of total crashes (7\%) in Kansas. This can be explained by the fact that Kansas has lot of rural roads, where the speed limit is high and in rural roads, laws are not strictly enforced, all of which might lead to a larger number of fatal pedestrian crashes.

\subsection{Statistical Analysis}

Statistical analysis is performed to determine if there is any dependency or relationship between different variables considered in this study. The test of independence analyzes the independency of two variables using Chi-square distribution. The test is also called contingency table test. The dependency between age group and gender, light condition, and days of week were analyzed and is shown in Table 2. The cross-tabulation of different age groups and gender represents that male pedestrian crashes contribute to the highest number of crashes irrespective of age groups and the difference of number of crashes between male and female pedestrians is large. But when an older pedestrian is considered it is seen that the gender difference is smaller compared to young and middle-aged pedestrians. The Chi-square value for this group is found 254.56. With degree of freedom of 2, the tabulated value is 5.991 for $95 \%$ confidence level. As this value is much lower than the calculated value, conclusion can be drawn that there is a strong inter-relationship between age groups and gender. Contingency table analysis between age groups and light condition reveal the fact that for the older pedestrian majority of crashes occur in daylight while for young and middle-aged pedestrians most of the crashes occur in the dark. The reason might be less exposure for older pedestrian during dark hours leading to less crashes. The chi-square value in this case is calculated as 1284.1 and the tabulated chi-square value is found to be 12.59 for six degrees of freedom. This leads to the rejection of null hypotheses as the calculated chi-square value is much higher than the ta- 
bulated value. So, we conclude that there is a strong relationship between age groups and light condition.

Dependency between age groups and days of week is tested. From the cross tabulation of age groups and days of week, it is found that most of the fatal crashes occur on Friday and Saturday for both young and middle-aged pedestrian, but older pedestrians are more vulnerable during weekdays. The reason might be those older pedestrians do not go out during weekend, which contributes to a smaller number of crashes during the weekend than weekdays. As younger pedestrians are found to be more vulnerable during weekends, it could be assumed that they were under the influence of alcohol. This variable (alcohol) was not analyzed in this study. The calculated chi-square value for this group (age groups and days of week) is 246.51. Comparing this value with the tabulated chi-square value, which is 21.03 for 12 degrees of freedom, leads to reject the null hypothesis and dependency between age groups and days of week is established.

Contingency table analysis between gender and speed limit (Table 3) gives a chi-square value of 358.32 , with four degrees of freedom; we get the calculated

Table 2. Contingency table between age and gender, light condition and days of week.

\begin{tabular}{|c|c|c|c|c|c|c|c|}
\hline \multirow{2}{*}{ Description } & \multicolumn{2}{|c|}{ Young } & \multicolumn{2}{|c|}{ Middle Aged } & \multicolumn{2}{|c|}{ Older } & \multirow{2}{*}{ Total } \\
\hline & Number & Percent & Number & Percent & Number & Percent & \\
\hline \multicolumn{8}{|l|}{ Gender } \\
\hline Female & 1758 & $33 \%$ & 4109 & $29 \%$ & 1906 & $59 \%$ & 7773 \\
\hline Male & 3652 & $67 \%$ & 10441 & $71 \%$ & 2789 & $41 \%$ & 16,862 \\
\hline Total & 5390 & $100 \%$ & 14,550 & $100 \%$ & 4695 & $100 \%$ & 24,635 \\
\hline & \multicolumn{7}{|c|}{ Chi-squared value $=254.56, \mathrm{df}=2$, Significant } \\
\hline \multicolumn{8}{|l|}{ Light Condition } \\
\hline Daylight & 1301 & $31 \%$ & 3274 & $22 \%$ & 2196 & $47 \%$ & 6771 \\
\hline Dark & 1527 & $36 \%$ & 5485 & $37 \%$ & 921 & $20 \%$ & 7933 \\
\hline Dark but lighted & 1184 & $28 \%$ & 5602 & $38 \%$ & 1326 & $28 \%$ & 8112 \\
\hline Dawn and Dusk & 191 & $5 \%$ & 505 & $3 \%$ & 227 & $5 \%$ & 923 \\
\hline Total & 4203 & $100 \%$ & 14,866 & $100 \%$ & 4670 & $100 \%$ & 23,739 \\
\hline & \multicolumn{7}{|c|}{ Chi-squared value $=1284, \mathrm{df}=6$, Significant } \\
\hline \multicolumn{8}{|l|}{ Day of Week } \\
\hline Sunday & 868 & $16 \%$ & 2318 & $16 \%$ & 432 & $9 \%$ & 3618 \\
\hline Monday & 633 & $12 \%$ & 1806 & $12 \%$ & 699 & $15 \%$ & 3138 \\
\hline Tuesday & 599 & $11 \%$ & 1819 & $12 \%$ & 739 & $16 \%$ & 3157 \\
\hline Wednesday & 652 & $12 \%$ & 1968 & $13 \%$ & 750 & $16 \%$ & 3370 \\
\hline Thursday & 655 & $13 \%$ & 1907 & $13 \%$ & 665 & $14 \%$ & 3227 \\
\hline Friday & 837 & $16 \%$ & 2359 & $16 \%$ & 785 & $17 \%$ & 3981 \\
\hline Saturday & 1039 & $20 \%$ & 2780 & $18 \%$ & 613 & $13 \%$ & 4432 \\
\hline Total & 5283 & $100 \%$ & 14,957 & $100 \%$ & 4683 & $100 \%$ & 24,923 \\
\hline
\end{tabular}


Table 3. Contingency table between gender and speed limit.

\begin{tabular}{cccccc}
\hline \multirow{2}{*}{ Description } & \multicolumn{2}{c}{ Male } & \multicolumn{2}{c}{ Female } & \multirow{2}{*}{ Total } \\
\cline { 2 - 4 } & Number & Percentage & Number & Percentage & \\
\hline Speed Limit & & & & & \\
30 mph or less & 2889 & $18 \%$ & 1845 & $27 \%$ & 4735 \\
35 or 40 mph & 4395 & $28 \%$ & 2124 & $31 \%$ & 6519 \\
45 or 50 mph & 3404 & $22 \%$ & 1317 & $19 \%$ & 4721 \\
55 mph & 2497 & $16 \%$ & 811 & $12 \%$ & 3308 \\
60 mph or higher & 2587 & $16 \%$ & 730 & $11 \%$ & 3317 \\
Total & 15,772 & $100 \%$ & 6827 & $100 \%$ & 22,599 \\
& \multicolumn{5}{c}{ Chi-squared value $=358.32, \mathrm{df}=4$, Significant } \\
\end{tabular}

chi-square value of 9.488. As the calculated value is much higher than the tabulated value, it can be said that there is a strong relationship between gender and speed limit. From the contingency table analysis, it can be concluded that male pedestrians tend to have strong association with higher speed limit than female pedestrians. Also, it was found that the lower the speed limit is, the stronger the association is with female pedestrians.

\subsection{Countermeasures}

Based on the findings, some of the countermeasures have been suggested which can minimize or lessen the number of pedestrian crashes.

The countermeasures have been categorized into three following sections [13]:

- Geometric countermeasures: Some of the geometric countermeasures that can be considered are converting unsignalized intersections into roundabout, installing pedestrian underpass/overpass, installing raised median at unsignalized intersections, installing Refuge Island, installing raised pedestrian crossing, providing paved shoulders etc.

- Signalized countermeasures: Signalized countermeasures can include installing exclusive pedestrian phasing, improving signal timing, replacing existing WALK/DON'T WALK signals with pedestrian countdown signal heads, removing unwarranted signals (one-way street), increasing pedestrian crossing time, implementing leading pedestrian interval etc.

- Signs, marking and operational countermeasures: Adding intersection lighting, adding segment lighting, improving pavement friction, prohibiting right turn on red, prohibiting left turns, advanced stop/yield sign, converting parallel lane into high visibility crosswalk, restricting parking near intersections etc. could be some of the measures that can be taken as operational countermeasures.

\section{Conclusions}

An attempt has been made in this study to identify the contributing factors of 
fatal pedestrian crashes both in Kansas and the USA and at the same time, comparison on the basis of contributing factors has been made between Kansas and the USA. Initially, the trends of fatal pedestrian crashes both in Kansas and the USA are identified. Conclusion is made by remarking that the percentage of pedestrian fatalities to that of total fatalities both for Kansas and USA does not reveal that the overall safety situation has been improved. It is found in the study that exposure level of walking has been decreased which might contribute to the lower number of fatal pedestrian crashes. The findings of the study can be summarized as follows:

- When involved persons in fatal pedestrian crashes are analyzed, it is found that male pedestrians contribute to the significantly larger number of fatal pedestrian crashes than female pedestrians both for Kansas and USA.

- Analysis of age groups reveals the fact that pedestrians aged between 45 and 54 , and 65+ years old are the most vulnerable groups among all age groups both for Kansas and USA. This finding is well consistent with a study done by Siddiqui et al. [5].

- When days of week of fatal pedestrian crashes are considered, it is found that Friday and Saturday have the largest number of fatal pedestrian crashes than any other days of the week.

- When time of occurrence is considered, it is found that fatal pedestrian crashes occur mostly during off peak hours of the day and especially, after 6 pm.

- Among different months analyzed, it is observed that November and December are the prime months for fatal pedestrian crashes to occur. For Kansas, March and August are also critical for fatal pedestrian crashes.

- When location is concerned, it is examined that most of the fatal pedestrian crashes occur in non-intersection, which is mid-block. Intersections have a very few pedestrian crashes both for Kansas and USA. The same result has also been obtained by a study done by Siddiqui et al. [5].

- Among different atmospheric conditions observed, it was found that clear atmospheric conditions lead to the larger number of fatal pedestrian crashes compared to adverse atmospheric condition. When light condition is studied, it is found that majority of fatal crashes occur in dark conditions, even if lighted. This result resembles the study done by Carter et al. [6] where dark hours are found to have association with fatal pedestrian crashes.

- Speed limits have the different results for Kansas and the USA. Speed limits higher than $60 \mathrm{mph}$ result in a larger number of fatal pedestrian crashes in Kansas (similar result has been obtained by a study done by Corben et al. [7]) whereas speed limits between 30 and $40 \mathrm{mph}$ contribute to the larger number of crashes in the USA.

The identified factors related to fatal pedestrian crashes are helpful to implement potential countermeasures which will ultimately reduce the number of fatal pedestrian crashes. 


\section{Acknowledgements}

The author would like to acknowledge the Kansas Department of Transportation for providing necessary data for carrying out this research.

\section{Conflicts of Interest}

The author declares no conflicts of interest regarding the publication of this paper.

\section{References}

[1] Federal Highway Administration, US Department of Transportation (2005) Traveler Opinion and Perception (TOP) Survey.

[2] Pucher, J. and Dijkstra, L. (2000) Making Walking and Cycling Safer: Lessons from Europe. Transportation Quarterly, 54, 25-50.

[3] National Highway Traffic Safety Administration (NHTSA) (2010) Traffic Safety Facts 2010. US Department of Transportation, Washington DC.

[4] Spainhour, L.K., Wootton, I.A., Sobanjo, J.O. and Brady, P.A. (2006) Causative Factors and Trends in Florida Pedestrian Crashes. Transportation Research Record: Journal of the Transportation Research Board, 1982, 90-98. https://doi.org/10.3141/1982-13

[5] Siddiqui, N.A., Chu, X. and Guttenplan, M. (2006) Crossing Locations, Light Conditions, and Pedestrian Injury Severity. Transportation Research Record: Journal of the Transportation Research Board, 1982, 141-149. https://doi.org/10.3141/1982-19

[6] Carter, D.L. and Council, F.M. (2007) Factors Contributing to Pedestrian and Bicycle Crashes on Rural Highways. In: 86th Annual Meeting of TRB, National Research Council, Washington DC.

[7] Corben, B. and D'Elia, A. (2007) Estimating Pedestrian Fatal Crash Risk. Publication of the Monash University Accident Research Centre.

[8] Kansas Department of Transportation (2010) Kansas Accident Reporting System (KARS) Database.

[9] National Highway Traffic Safety Administration, US Department of Transportation (2011) Fatality Accident Report System.

https://www.nhtsa.gov/research-data/fatality-analysis-reporting-system-fars

[10] Goswami, A. and Sonowal, R. (2009) A Statistical Analysis of Road Traffic Accidents in Dibrugarh City, Assam, India. Division of Epidemiology and Nutrition, Regional Medical Research Centre, Dibrugarh.

[11] Navidi, W. (2011) Statistics for Engineers and Scientist. McGraw-Hill, New York.

[12] Pucher, J. and Renne, J.L. (2003) Socioeconomics of Urban Travel: Evidence from the 2001 NHTS. Transportation Quarterly, 57, 49-78.

[13] Federal Highway Administration (FHWA) Report (2018) Toolbox of Pedestrian Countermeasures and Their Potential Effectiveness. 